\title{
Evaluation of Predictive Values of Naples Prognostic Score in Patients with Unresectable Stage III Non-Small Cell Lung Cancer
}

\author{
Dong Guo $\mathbb{D}^{\prime}$ \\ Jiafeng $\mathrm{Liu}^{2}$ \\ Yanping $\mathrm{Li}^{3}$ \\ Chao $\mathrm{Li}^{3}$ \\ Quan $\mathrm{Liu}^{3}$ \\ Shengjun Ji $\mathbb{I D}^{4}$ \\ Shuchai Zhu' \\ 'Department of Radiation Oncology, \\ Fourth Hospital of Hebei Medical \\ University, Shijiazhuang, People's Republic \\ of China; ${ }^{2}$ Department of Radiotherapy, \\ Rizhao Center Hospital, Rizhao, People's \\ Republic of China; ${ }^{3}$ Sunshine Union \\ Hospital, Weifang, People's Republic of \\ China; ${ }^{4}$ Department of Radiotherapy \& \\ Oncology, The Affiliated Suzhou Hospital \\ of Nanjing Medical University, Suzhou, \\ People's Republic of China
}

Background: Naples prognosis score (NPS) is a new prognostic score according to host inflammatory and nutritional state, and it could be useful for predicting tumor prognosis based on albumin level, total cholesterol level, neutrophil-to-lymphocyte ratio, and lymphocyte-to-monocyte ratio. This study aimed to evaluate the clinical significance of Naples prognostic score (NPS) in stage III non-small cell lung cancer patients (NSCLC).

Patients and Methods: In this study, 206 patients diagnosed with locally advanced NCCLC receiving chemoradiotherapy were retrospectively reviewed from January 2013 to January 2017. The included patients were divided into 3 groups according to NPS (group 0, group 1, and group 2), and the associations of the NPS with clinical characteristics and outcomes were evaluated among the groups. Survival curves for the NPS were analyzed using the Kaplan-Meier method. Univariate and multivariate analyses were performed using the Cox proportional hazards regression model to evaluate the prognostic value of overall survival (OS) and progression-free survival (PFS).

Results: The median follow-up time of this study was 37.0 (range, 13-59) months. The median OS was 27 months in group 0, 23 months in group 1, and 21 months in group 2, and median PFS was 15, 12 and 13 in group 0, group 1 and group 2, respectively. Age was significantly different among the 3 groups. The NPS was superior to other host inflammatory and nutritional indexes for prognostic risk stratification. In the multivariate analysis, NPS was identified as an independent prognostic indicator for OS and PFS (all $P<0.05$ ).

Conclusion: The NPS system is considered to be a useful predictor of outcomes in patients with stage III NSCLC.

Keywords: Naples prognostic score, stage III NSCLC, predictive values

\section{Introduction}

Lung cancer is one of the most common malignant tumors and the leading cause of death among cancers worldwide. ${ }^{1}$ Non-small cell lung cancer (NSCLC) compromises more than $80 \%$ of all lung cancers cases, whereby $30-40 \%$ of these patients are diagnosed with stage III NSCLC. ${ }^{2}$ The majority of stage III NSCLC are not suitable for surgical resection due to serious internal medicine disease, poor excised completely or extensive lymph node metastases. Chemoradiotherapy is the standard of care for patients with unresectable stage III NSCLC tumors, which are generally treated with 40-50 days of definitive concurrent chemoradiotherapy, and the option of additional consolidation chemotherapy for patients with high tumor load. ${ }^{3}$ Despite advances in antineoplastic drugs and radiotherapy, stage III patients still
Correspondence: Shengjun Ji; Shuchai

Zhu

Email drshengjunji@I63.com;

3562069484@qq.com 
have locoregional failure rates of $35-70 \%$, and 5-overall survival (OS) rates are $10-20 \%,{ }^{4-6}$ with median OS of 10 14 months. $^{7}$ The clinical outcomes could be different for stage III NSCLC patients treated with the same therapy due to individual heterogeneity, which is the main clinical challenge in the management strategy of cancer patients. Therefore, identification of prognostic factors to categorize risk levels is crucial for individualized precision therapy.

Increasing evidence confirmed that systemic inflammation exerts a vital biological role during malignant tumors proliferation, metastasis, invasion, and angiogenesis. ${ }^{8-10}$ Systemic inflammation molecules affect biochemical mechanisms where tumor cells secrete inflammatory response cytokines, potentially reflecting degree of biological reactivity of tumor cells. The inflammation-related prognostic indicators, including neutrophil-lymphocyte ratio (NLR), lymphocyte-monocyte ratio (LMR) and platelet-lymphocyte ratio (PLR), could be used as predictors of the prognosis in NSCLC. ${ }^{11,12}$ However, these predictors also remained deficient for the fully reflecting prognosis and patients' whole condition by just one single inflammation-related indicator. Prognostic indicators reflecting host nutritional or immunological status, such as the prognostic nutritional index (PNI), systemic immune-inflammatory index (SII) and controlling nutritional status (CONUT), are associated with tumor prognosis. ${ }^{13-16}$ Therefore, an increasing number of clinical risk prognostic models, including inflammation-related and host nutritional status indicators, are urgently needed.

The Naples prognostic score (NPS), which is a new prognostic scoring system based on albumin, total cholesterol concentration, NLR, and LMR, comprehensively reflects the patient's inflammation and nutritional status. Researchers have shown its predictive value in endometrial cancer, ${ }^{17}$ colorectal cancer $^{18}$ and esophageal squamous cell carcinoma. ${ }^{19}$ In addition, the predictive value of NPS is more accurate than that of other inflammatoryrelated and nutritional-related scoring systems. ${ }^{20}$ NPS was evaluated in association with the cancer prognosis for both inflammation and nutritional status, and it has a better prognostic performance than either of them alone. However, the significance of NPS in the prognosis of patients with unresectable stage III NSCLC remains largely unknown. As a result, the present study had two aims: first, to evaluate the prognostic value of NPS among stage III NSCLC patients, second, to investigate the associations of the NPS with clinical characteristics.

\section{Patients and Methods}

\section{Study Population}

From January 2013 to January 2017, this study assessed the 206 patients undergoing chemoradiotherapy due to unresectable stage III NSCLC at Rizhao Center Hospital. The inclusion criteria were as follows: histologically or cytologically diagnosed with confirmed stage III NSCLC; Karnofsky performance score (KPS) 70-100; no prior anti-tumor treatment; availability of complete laboratory results; followed up for over 1 year. Patients who met the following criteria were excluded from the retrospective study: had inflammatory disorder or infectious disease; had malignant tumors at other sites or serious diseases affecting immune and the nutritional status. This retrospective cohort study was approved by the institutional review board of Rizhao Center Hospital institutions. All patients were provided with written informed consent. The study was performed in accordance with the International Conference on Harmonisation Guidelines on Good Clinical Practice and the Declaration of Helsinki.

\section{Treatment Regimes}

The standard chemoradiotherapy in our study consists of 6-7 weeks of intensity-modulated radiation therapy (IMRT) with cisplatin-based chemotherapy. All enrolled patients received chest computed tomography (CT) scans before radiation therapy. Individual radiotherapy target planning is implemented in conjunction with positron emission tomography-CT (PET-CT) and chest enhanced CT imaging results. The IMRT dose was 60 to $66 \mathrm{~Gy}$ in 1.8 to 2.0 Gy daily fractions, with 5 fractions a week. The radiotherapy plan was assessed and approved independently by 2 radiotherapy doctors (F.J and HL.P). In this study, one hundred and twenty $(120 / 206,58.3 \%)$ patients received cisplatin/docetaxel and eighty-six (86/206, 41.7\%) patients received cisplatin/etoposide.

\section{NPS System}

Routine laboratory blood test was performed, and the results were acquired from the Rizhao Center Hospital (Rizhao, China) within one week before IMRT. Based on the clinical information system, the following information was extracted from each study patient: gender, age, smoking status, pathological type, differentiation, tumor site, primary tumor size, TNM stage, serum albumin concentration, total cholesterol content, lymphocyte count, neutrophil count and monocytes count. As previously 
reported by Galizia et al, ${ }^{18}$ NPS was calculated based on the serum albumin concentration, total cholesterol content, NLR and LMR. For patients with serum albumin $\geq 4 \mathrm{mg} / \mathrm{dL}$, total cholesterol content $>180 \mathrm{mg} / \mathrm{dL}$, NLR < 2.96 , or LMR $>4.44$ were scored as 0 , while patients with serum albumin $<4 \mathrm{mg} / \mathrm{dL}$, total cholesterol content $\leq 180 \mathrm{mg} / \mathrm{dL}, \mathrm{NLR} \geq 2.96$, or $\mathrm{LMR} \leq 4.44$ was scored as 1. Patients were categorized into three groups based on NPS system: patients with a score of 0,1 or 2 , and 3 or 4 were assigned to groups 0,1 , and 2, respectively. And NPS were further analyzed in subgroups of stage IIIA or stage IIIB.

\section{Follow-Up}

Progression-free survival (PFS) was defined as the time from the date of starting radiotherapy to the date of disease progression recurrence, and OS was defined as the time between radiotherapy and death from any cause. Patients were followed up every 3 months for the first 3 years, then every 6 months for the following years and annually thereafter. Patient physical examination, laboratory test, CT or PET-CT were performed for evaluation of primary tumor and distant metastasis. Follow-up was conducted on 206 patients until August 2018.

\section{Statistical Analysis}

The IBM SPSS Statistics 23.0 (SPSS, Inc., Chicago, IL, USA) and GraphPad Prism software (version 5.0) were used to perform statistical analyses. $T$-tests or chi-square tests were applied to analyze the correlations between categorical variables and categorical data and NPS. Receiver operating characteristic (ROC) curves were generated to evaluate the recurrence prediction ability and differences of the scoring systems. The survival curves were performed by Kaplan-Meier method, and Log rank test was applied to compare prognostic survival differences among the NPS groups. The significant variables selected from univariate analysis were incorporated into multivariate analysis according to the Cox proportional hazards regression model. All the variables identified with a $P<0.05$ were indicated to have statistical significance.

\section{Results}

\section{Patient Characteristics}

A total of 206 patients were diagnosed with NSCLC of unresectable stage IIIA or IIIB and received chemoradiotherapy in this study from January 2013 to January 2017 (Figure 1). The study population consisted of 86 females

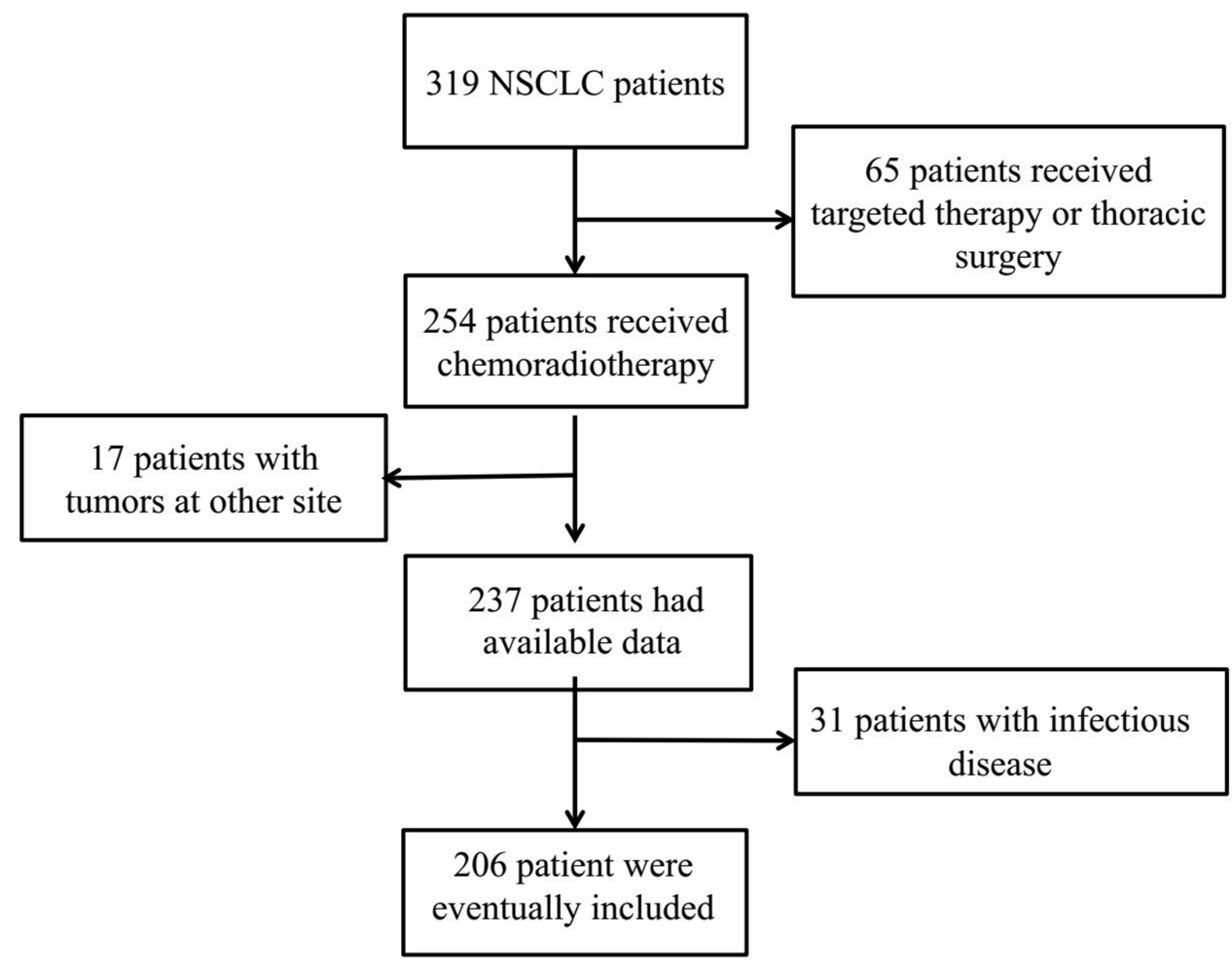

Figure I The flow chart of case screening. 
(41.7\%) and 120 male (58.3\%), with the median age of 62 (range, 36-84) years. With regard to the tumor location, 96 $(46.60 \%)$ tumors were located in the left and $110(53.4 \%)$ tumors occurred in the right. Eighty-one (39.3\%) patients had stage IIIA, and $125(60.7 \%)$ had stage IIIB. One hundred and nineteen $(57.8 \%)$ patients suffered recurrence and metastasis, and $138(70.0 \%)$ patients died. In total, the median follow-up time was 37 months and ranged from 13 months to 59 months.

\section{Associations of NPS System with Clinicopathologic Features}

Based on the NPS system, the three groups were as follows: Group 0, 32 (15.5\%) patients; Group 1, 135 (65.5\%) patients; and Group 2, 39 (19.0\%) patients (Table 1). Age $(P=0.002)$ was significantly different among the three NPS groups. However, there was no significant relation in patient, KPS, patient histology, differentiation, TNM status, serum CEA or CYFRA21-1 among the three groups.

\section{NPS System Parameter and ROC Curves for Recurrence Prediction}

All of the peripheral blood indicators are summarized in Table 2. The patients had a median serum albumin of 4.50 (95\% confidence interval [CI]: 4.00-5.00), total cholesterol of 240 (95\% CI: 230.00-261.99), NLR of 3.71 (95\% CI: 3.55-3.85) and LMR of 2.97 (95\% CI: $2.78-$ 3.32). The scatter diagrams of serum albumin, total cholesterol, NLR and LMR were shown in Figure 2. NPS was found to have the largest area under the curve (AUC $=0.715 ; 95 \%$ CI, 0.647-0.783; $P<0.001$ ). The AUCs were $0.589(P=0.029), 0.623 \quad(P=0.003), 0.630$ $(P=0.001)$, and $0.566(P=0.105)$ for serum albumin, total cholesterol, NLR and LMR, respectively.

\section{Univariate Analysis of OS and PFS}

The univariate analysis results revealed that KPS ( $P=0.001)$, differentiation $(P=0.002)$, primary tumor size $(P=0.012), \quad$ cTNM status $(P=0.001)$, response rate $(P=0.022)$ and NPS $(P<0.001)$ were significantly correlated with OS. KPS $(P=0.005)$, differentiation $(P=0.003)$, cTNM status $(P=0.004)$, response rate $(P=0.006)$ and NPS $(P<0.001)$ were significantly correlated with PFS (Table 3). We found no significant association between OS or PFS and age, patient histology, tumor site, serum CEA, and CYFRA21-1 (all $P>0.05$ ). Kaplan-Meier analyses were used to compare serum albumin, total cholesterol, NLR, LMR and NPS. As shown in Figure 3, patients with serum albumin $\geq 4 \mathrm{mg} / \mathrm{dL}$, total cholesterol $>180 \mathrm{mg} / \mathrm{dL}$ and NLR $\leq 2.96$ had significantly longer OS. Regarding PFS, the patients with serum albumin $<4 \mathrm{mg} /$ $\mathrm{dL}$, total cholesterol $\leq 180 \mathrm{mg} / \mathrm{dL}$ and NLR $>2.96 \mathrm{had}$ significantly worse PFS (Figure 4). The median OS in patients with group 0 was significantly higher than the OS in patients with group 1 and group 2 ( 27 vs 23 vs 21 months; $P<0.001$ ) (Figure 5A). Similarly, the median PFS were 15,12 , and 13 months for patients with group 0,1 , and 2 , respectively $(P<0.001)$ (Figure $5 \mathrm{~B})$. Further subgroup analysis revealed that patients with high NPS were more likely to have poorer outcomes in stage IIIA (OS, $P<0.001$; PFS, $P<0.001$ ) and stage IIIB (OS, $P=0.001$; PFS, $P=0.004$ ) (Figure 6).

\section{Multivariate Analysis of OS and PFS}

To future investigate the prediction value of NPS in stage III NSCLC, multivariate analysis results are shown in Table 4. Data confirmed that NPS was independent factor of OS (hazard ratio [HR], 1.988; 95\% CI, 1.326-2.981; $P=0.001)$ and that NPS (HR $=1.793,95 \%$ CI: $1.182-2.721$, $P=0.006$ ) was significant prognostic factors of PFS. We also found that TNM status was as an independent predictor in $\mathrm{OS}(P=0.031)$.

\section{Discussion}

Establishment of a reliable prognostic prediction system is urgent in indicating risk stratification and tailoring appropriate treatment strategies for stage III NSCLC. The analysis of systemic inflammation and nutritional status can offer a method to evaluate the association between them and outcomes. The NPS as a new prognostic scoring system reflecting the patient's inflammation and nutritional status, to the best of our knowledge, the present study was the first to demonstrate the first association of NPS with outcomes and NPS serve better accuracy compared with other prognostic scoring systems.

In 1863, Virchow was the first researcher to report the relationship between inflammation and tumors. ${ }^{21}$ Then, many researchers investigate peripheral blood inflammation cells and explore the intrinsic relationship between inflammation and cancer. Inflammation is known to be an important factor in tumor progression and is one of the recognized hallmarks of tumor. ${ }^{22}$ In addition to, inflammatory cells are involved in tumor angiogenesis, immunosuppressive, resistance of radiation and chemotherapy drug by secreting inflammatory cytokines and immune negative 
Table I Association of the Naples Prognostic Score and Clinical Characteristics

\begin{tabular}{|c|c|c|c|c|c|}
\hline \multirow[t]{2}{*}{ Characteristics } & \multirow[b]{2}{*}{ Values $(\mathrm{N}=206)$} & \multicolumn{3}{|c|}{ Naples Prognostic Score Group } & \multirow[t]{2}{*}{ P-value } \\
\hline & & Group $0(\mathrm{~N}=32)$ & Group I $(N=135)$ & Group 2 (N=39) & \\
\hline Age (years) & & & & & 0.002 \\
\hline$<60$ & 87 & 13 & 48 & 26 & \\
\hline$\geq 60$ & 119 & 19 & 87 & 13 & \\
\hline Gender & & & & & 0.524 \\
\hline Male & 120 & 21 & 75 & 24 & \\
\hline Female & 86 & 11 & 60 & 15 & \\
\hline Smoke & & & & & 0.302 \\
\hline No & III & 15 & 78 & 18 & \\
\hline Yes & 95 & 17 & 57 & 21 & \\
\hline KPS & & & & & 0.219 \\
\hline $90-100$ & 92 & 17 & 62 & 13 & \\
\hline $70-80$ & 114 & 15 & 73 & 26 & \\
\hline Patient histology & & & & & 0.458 \\
\hline SCC & 90 & II & 60 & 19 & \\
\hline$A D$ & 116 & 21 & 75 & 20 & \\
\hline Differentiation & & & & & 0.076 \\
\hline Well/Moderate & 79 & 15 & 55 & 9 & \\
\hline Poor & 127 & 17 & 80 & 30 & \\
\hline Tumor site & & & & & 0.182 \\
\hline Left & 96 & 16 & 67 & 13 & \\
\hline Right & 110 & 16 & 68 & 26 & \\
\hline Primary tumor size & & & & & 0.050 \\
\hline$\leq 4 \mathrm{~cm}$ & 104 & 16 & 75 & 13 & \\
\hline$>4 \mathrm{~cm}$ & 102 & 16 & 60 & 26 & \\
\hline cTNM status & & & & & 0.256 \\
\hline IIIA & 81 & 16 & 53 & 12 & \\
\hline IIIB & 125 & 16 & 82 & 27 & \\
\hline Serum CEA(ng/mL) & & & & & 0.280 \\
\hline Normal & 130 & 23 & 80 & 27 & \\
\hline High & 76 & 9 & 55 & 12 & \\
\hline CYFRA2I-I (ng/mL) & & & & & 0.585 \\
\hline Normal & 123 & 18 & 84 & 21 & \\
\hline High & 83 & 14 & 51 & 18 & \\
\hline Treatment modality & & & & & 0.960 \\
\hline CCRT & 93 & 15 & 60 & 18 & \\
\hline SCRT & 113 & 17 & 75 & 21 & \\
\hline Response rate & & & & & 0.251 \\
\hline$C R+P R$ & 79 & II & 57 & II & \\
\hline$S D+P D$ & 127 & 21 & 78 & 28 & \\
\hline
\end{tabular}

Abbreviations: KPS, Karnofsky performance score; SCC, squamous cell carcinoma; AD, adenocarcinoma; BM, brain metastases; CEA, carcinoembryonic antigen; CYFRA2I-I, cytokeratin 19 fragments; CCRT, concurrent chemoradiotherapy; SCRT, Sequential chemoradiotherapy; CR, complete response; PR, partial response; SD, Stable disease; PD, progressive disease. 
Table 2 The Result of NPS System Parameter

\begin{tabular}{|l|c|}
\hline Parameters & Median (Range) \\
\hline Serum albumin $(\mathrm{mg} / \mathrm{dL})$ & $4.50(3.10-5.70)$ \\
Total cholesterol $(\mathrm{mg} / \mathrm{dL})$ & $240(\mathrm{II} .25-895.00)$ \\
Neutrophil & $6.19(2.16-12.19)$ \\
Lymphocyte & $1.72(0.45-4.85)$ \\
Monocyte & $0.54(0.14-4.90)$ \\
NLR & $3.7 \mathrm{I}(0.74-14.34)$ \\
LMR & $2.97(0.99-11.70)$ \\
\hline
\end{tabular}

Abbreviations: NLR, neutrophil-to-lymphocyte ratio; LMR, lymphocyte-tomonocyte ratio.

regulation of cell infiltration. ${ }^{23}$ Neutrophilia is an independent predictor of worse clinical outcome in malignant tumor patients. ${ }^{24,25}$ Lymphocytes act as the dominant antitumor cells. It has been demonstrated that lymphopenia is related to poor response and outcomes in several malignancies, including NSCLC. ${ }^{26-28} \mathrm{~A}$ high density of absolute monocyte counts is associated with shorter time to response to immune checkpoint inhibitors ${ }^{29,30}$ Basic research theories have been proposed to confirm this relationship. Neutrophils have several main functions: secreting growth factors to promote tumor formation; stimulating proliferation and metastasis by releasing inflammatory mediators; and protecting CTCs from immune cells attack. ${ }^{31,32}$ Lymphocytes participate in immune surveillance and kill tumor cells. ${ }^{33,34}$ Monocytes stimulate tumor angiogenesis, inflammatory response and improve tumor microenvironment by secreting oncostatin$\mathrm{M}$ and VEGF. ${ }^{35}$ NLR and LMR are more objective markers that reflect the inflammatory and immune status of the host. NLR was identified as predictive markers of clinical outcome in patients with various malignant tumors. ${ }^{36-38}$ There were also a number of clinical studies that investigated the prognostic significance of LMR, and the results confirmed that LMR was a significant prognostic predictor. $^{39,40}$
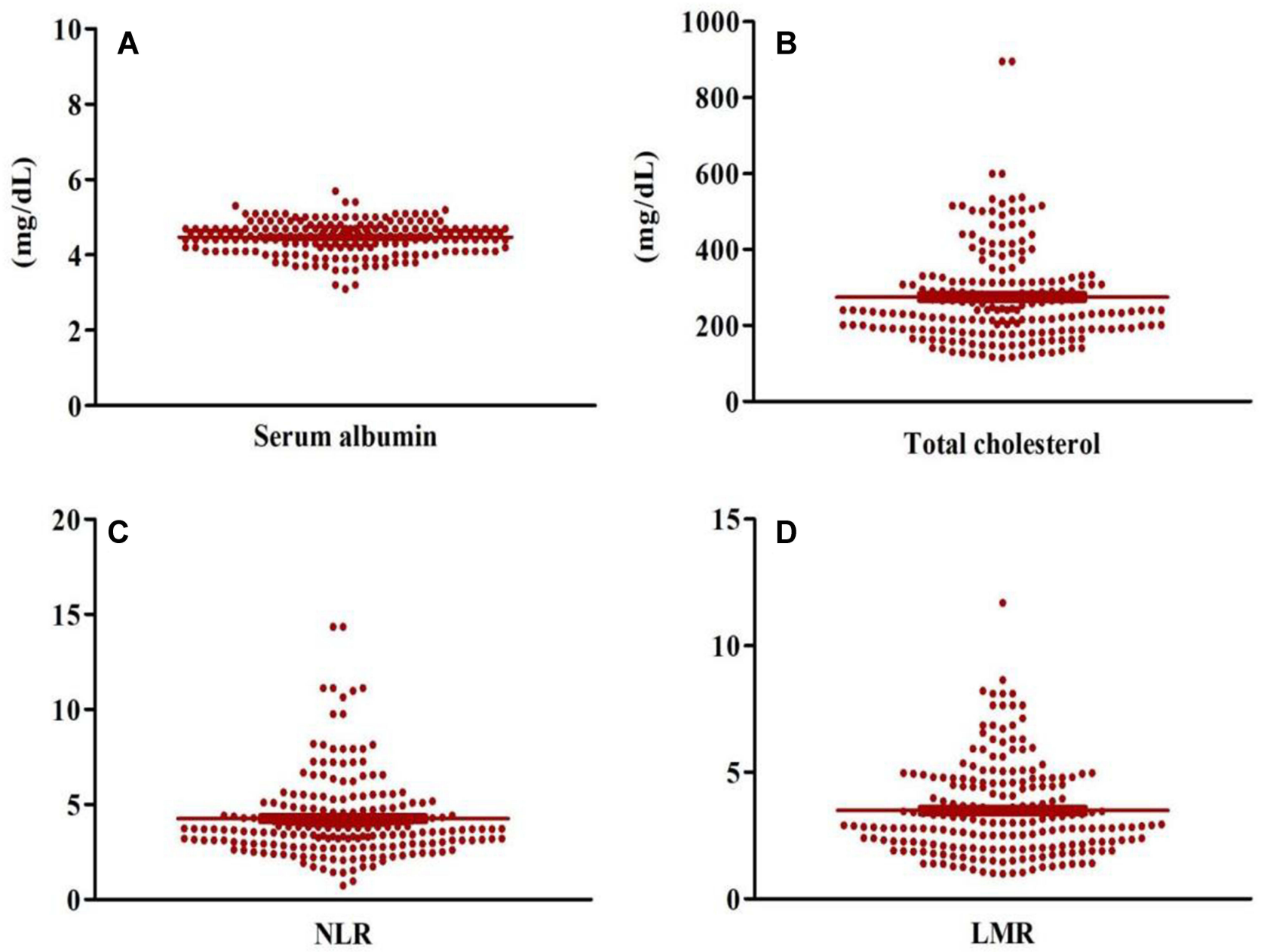

Figure 2 The expression levels of serum albumin (A), total cholesterol (B), NLR (C) and LMR (D) in scatter diagrams. 
Table 3 Univariate Cox Regression Analysis of OS and PFS

\begin{tabular}{|c|c|c|c|c|c|c|}
\hline \multirow[t]{2}{*}{ Characteristics } & \multicolumn{3}{|c|}{ os } & \multicolumn{3}{|c|}{ PFS } \\
\hline & HR & $95 \% \mathrm{Cl}$ & P-value & HR & $95 \% \mathrm{Cl}$ & P-value \\
\hline Age (years) & & & 0.096 & & & 0.481 \\
\hline$<60$ & 1.000 & & & 1.0000 & & \\
\hline$\geq 60$ & 0.747 & $0.530-1.053$ & & 0.875 & $0.605-1.267$ & \\
\hline Gender & & & 0.809 & & & 0.994 \\
\hline Male & 1.000 & $0.744-1.462$ & & 1.000 & $0.692-|.44|$ & \\
\hline Female & 1.043 & & & 0.998 & & \\
\hline Smoke & & & 0.099 & & & 0.071 \\
\hline No & 1.000 & $0.535-1.055$ & & 1.000 & $0.497-1.029$ & \\
\hline Yes & 0.751 & & & 0.715 & & \\
\hline KPS & & & 0.001 & & & 0.005 \\
\hline $90-100$ & 1.000 & $1.245-2.512$ & & 1.000 & $1.184-2.530$ & \\
\hline $70-80$ & 1.768 & & & 1.731 & & \\
\hline Patient histology & & & 0.894 & & & 0.553 \\
\hline SCC & 1.000 & $0.730-1.433$ & & 1.000 & $0.774-1.613$ & \\
\hline$A D$ & 1.023 & & & 1.117 & & \\
\hline Differentiation & & & 0.002 & & & 0.003 \\
\hline Well/Moderate & 1.000 & $1.252-2.647$ & & 1.000 & $1.235-2.784$ & \\
\hline Poor & 1.820 & & & 1.854 & & \\
\hline Tumor site & & & 0.111 & & & 0.248 \\
\hline Left & 1.000 & $0.939-1.848$ & & 1.000 & $0.86 I-1.785$ & \\
\hline Right & 1.317 & & & 1.240 & & \\
\hline Primary tumor size & & & 0.012 & & & 0.179 \\
\hline$\leq 4 \mathrm{~cm}$ & 1.000 & $1.102-2.183$ & & 1.000 & $0.893-|.84|$ & \\
\hline$>4 \mathrm{~cm}$ & $1.55 \mathrm{I}$ & & & 1.282 & & \\
\hline cTNM status & & & 0.001 & & & 0.004 \\
\hline IIIA & 1.000 & $1.291-2.654$ & & 1.000 & $1.209-2.640$ & \\
\hline IIIB & $1.85 \mathrm{I}$ & & & 1.786 & & \\
\hline Serum CEA(ng/mL) & & & 0.660 & & & 0.979 \\
\hline Normal & 1.000 & $0.765-1.526$ & & 1.000 & $0.691-1.462$ & \\
\hline High & 1.081 & & & 1.005 & & \\
\hline CYFRA2I-I (ng/mL) & & & 0.20 & & & 0.08 \\
\hline Normal & 1.000 & $0.560-1.129$ & 0 & 1.000 & $0.493-1.042$ & I \\
\hline High & 0.795 & & & 0.717 & & \\
\hline Treatment modality & & & 0.11 & & & 0.13 \\
\hline CCRT & 1.000 & $0.933-1.843$ & 8 & 1.000 & $0.914-1.909$ & 8 \\
\hline SCRT & 1.311 & & & 1.321 & & \\
\hline Response rate & & & 0.022 & & & 0.006 \\
\hline$C R+P R$ & 1.000 & $1.064-2.190$ & & 1.000 & I. $174-2.607$ & \\
\hline$S D+P D$ & 1.526 & & & 1.749 & & \\
\hline NPS Group & & & $<0.001$ & & & $<0.001$ \\
\hline 0,1 & 1.000 & 1.845 & & 1.000 & 1.600 & \\
\hline 2 & 2.691 & 3.925 & & 2.356 & 3.468 & \\
\hline
\end{tabular}

Abbreviations: OS, overall survival; PFS, progression-free survival; $\mathrm{HR}$, hazard ratio; $\mathrm{Cl}$, confidence interval; KPS, Karnofsky performance score; SCC, squamous cell carcinoma; AD, adenocarcinoma; CEA, carcinoembryonic antigen; CYFRA2I-I, cytokeratin 19 fragments; CCRT, concurrent chemoradiotherapy; SCRT, sequential chemoradiotherapy; CR, complete response; PR, partial response; SD, stable disease; PD, progressive disease. 

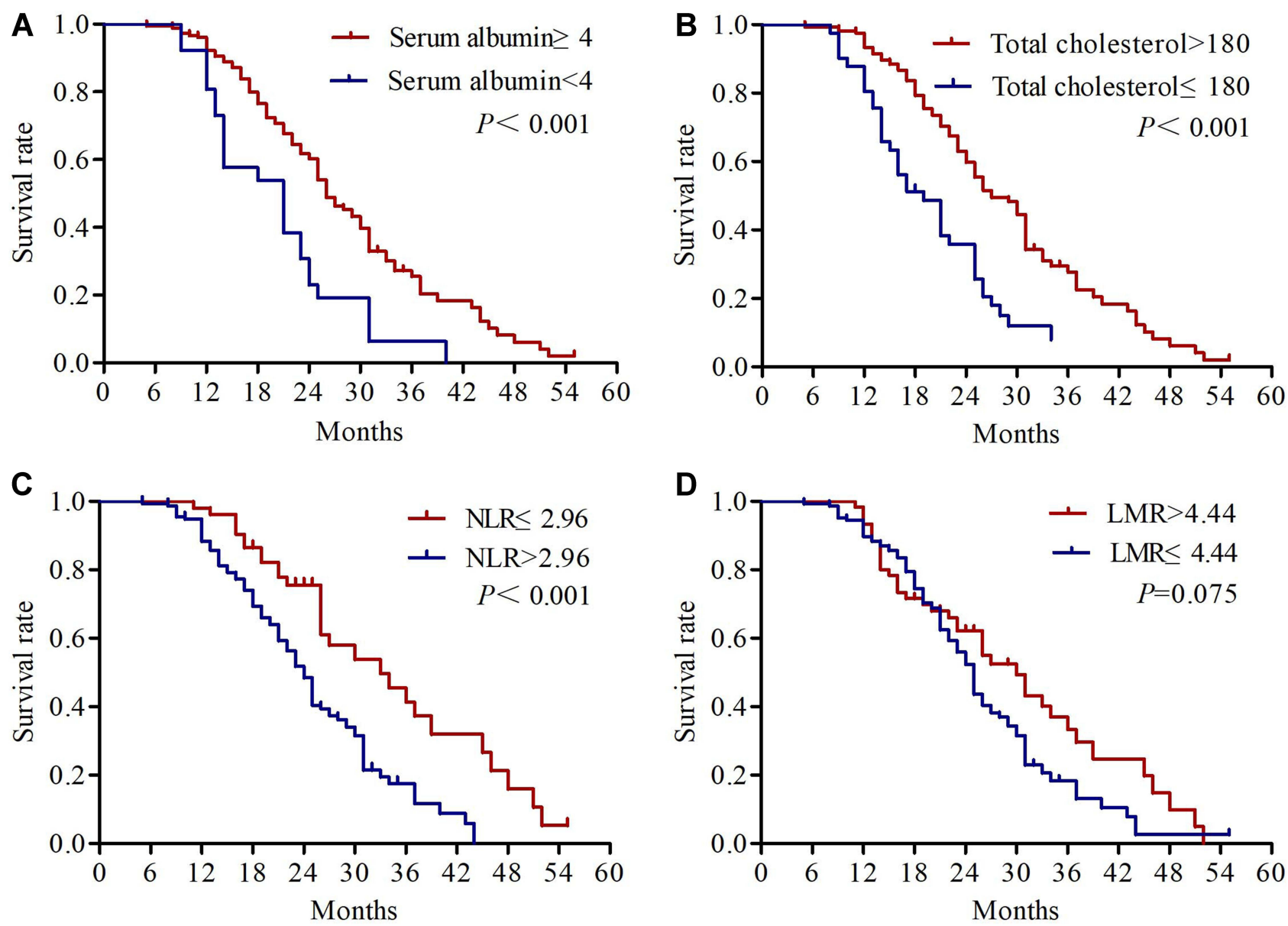

Figure 3 Kaplan-Meier survival curves of overall survival for serum albumin (A), total cholesterol (B), NLR (C) and LMR (D).

In addition to NLR and LMR, the biochemical mechanism by which NPS affects patient outcomes is also dependent on serum albumin, total cholesterol. Nutrition is closely associated with tumor growth, tumor angiogenesis and tumor progression and malnutrition is often the cause of these adverse factors. It is an urgent problem that we are faced with to search a prognostic evaluation system based on nutritional index. Serum albumin concentration is a marker for nutrition, and hypoalbuminemia has been proven to be a poor outcome in many nutritional scoring systems. ${ }^{41,42}$ Combined with the previous albumin-based prognostic evaluation system, we found that low albumin levels often represent highly malignant disease. In addition, total cholesterol content may be an important prognostic factor for overall survival in many human tumors, including NSLCL. ${ }^{43-45}$ The mechanism may be related to the following aspects: participating in the key malignant transformation of cellular signaling pathways via angiogenesis; inhibiting transmembrane signals transmission; loss of immune surveillance and immune attack capability in immune cells. ${ }^{46,47}$

Based on the above theoretical mechanism, NPS was based on four types of indicators, and NPS should be a more objective marker that reflects the patient's inflammation and nutritional status than all the other systemic inflammation scores. As previously analyzed, NPS had been confirmed as a predictive indicator for OS and had better predictive value than clinical prognostic parameters alone in patients with resected pancreatic cancer. ${ }^{48}$ Kano et al found that NPS group had significant association with histopathological response to neoadjuvant treatments, and high NPS was associated with poor prognosis in locally advanced esophageal cancer patients. ${ }^{49}$ In a similar study, Gennaro et al found that NPS is predictive of prognosis in colorectal cancer patients and may have important clinical guiding effects. ${ }^{18}$ Moreover, high NPS may be a significant contributing factor to poor prognosis in 

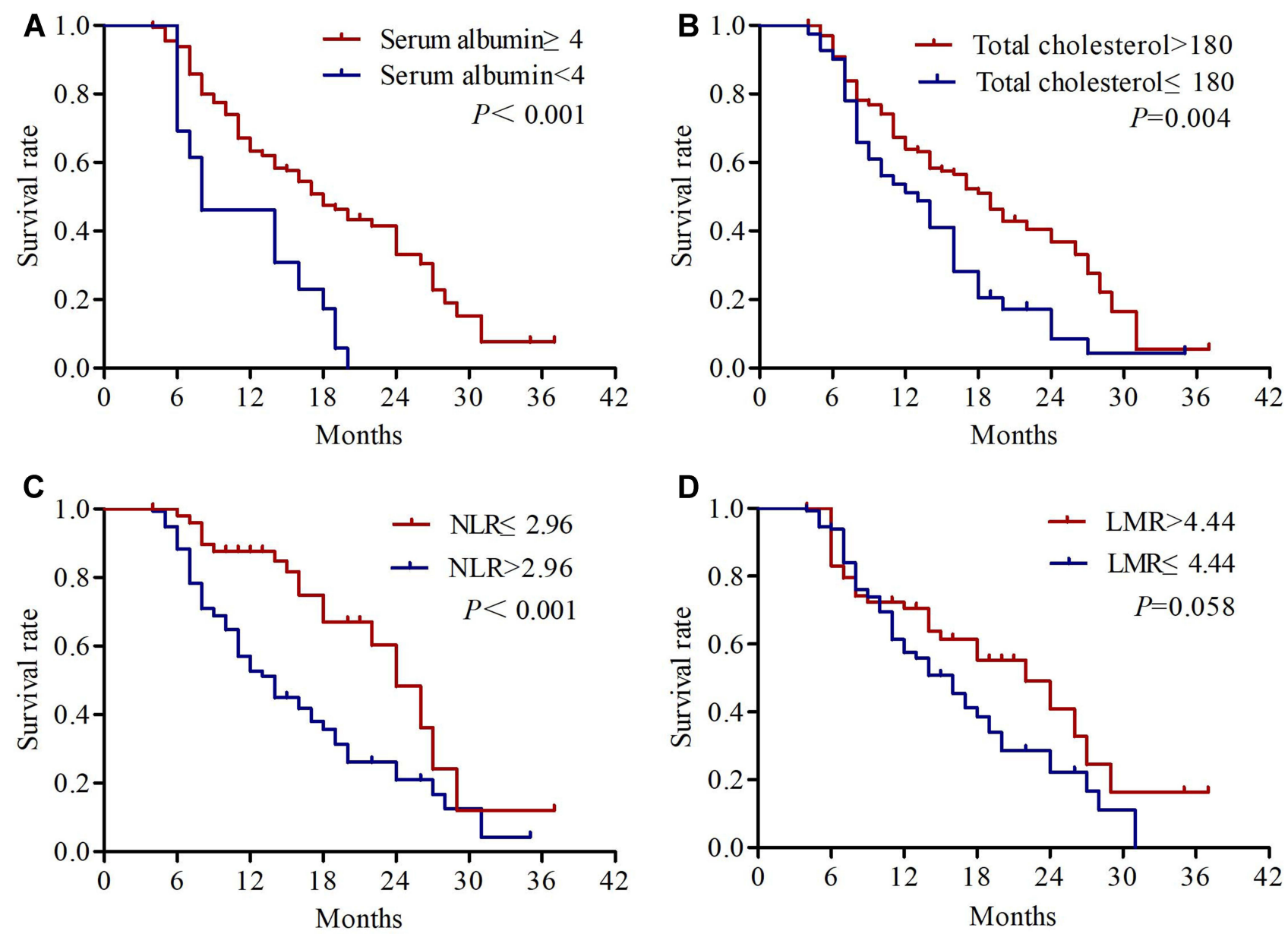

Figure 4 Kaplan-Meier survival curves of progression-free survival for serum albumin (A), total cholesterol (B), NLR (C) and LMR (D).
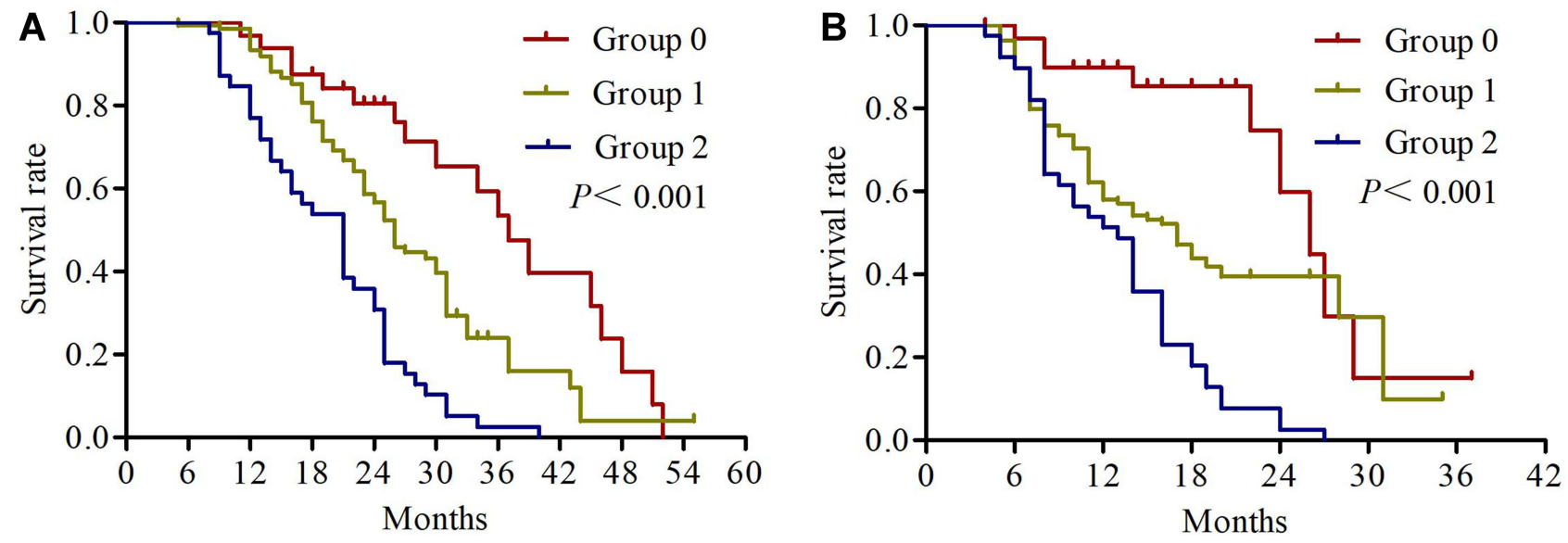

Figure 5 Kaplan-Meier survival curves of overall survival (A) and progression-free survival (B) for NPS group.

endometrial cancer, which is a valuable significantly independent prognostic factor. ${ }^{17}$ However, clinical data on NPS score system for NSCLC are still lacking, particularly in locally advanced NSCLC, which has not been reported so far. Li et al conducted a study involving 445 patients with early-stage NSCLC in a single-center prospectively maintained database. ${ }^{50}$ In this study, OS and DFS were significantly shortened in high NPS score group and preoperative NPS could be an independent prognostic indicator. In addition, NPS held the better discriminative value 

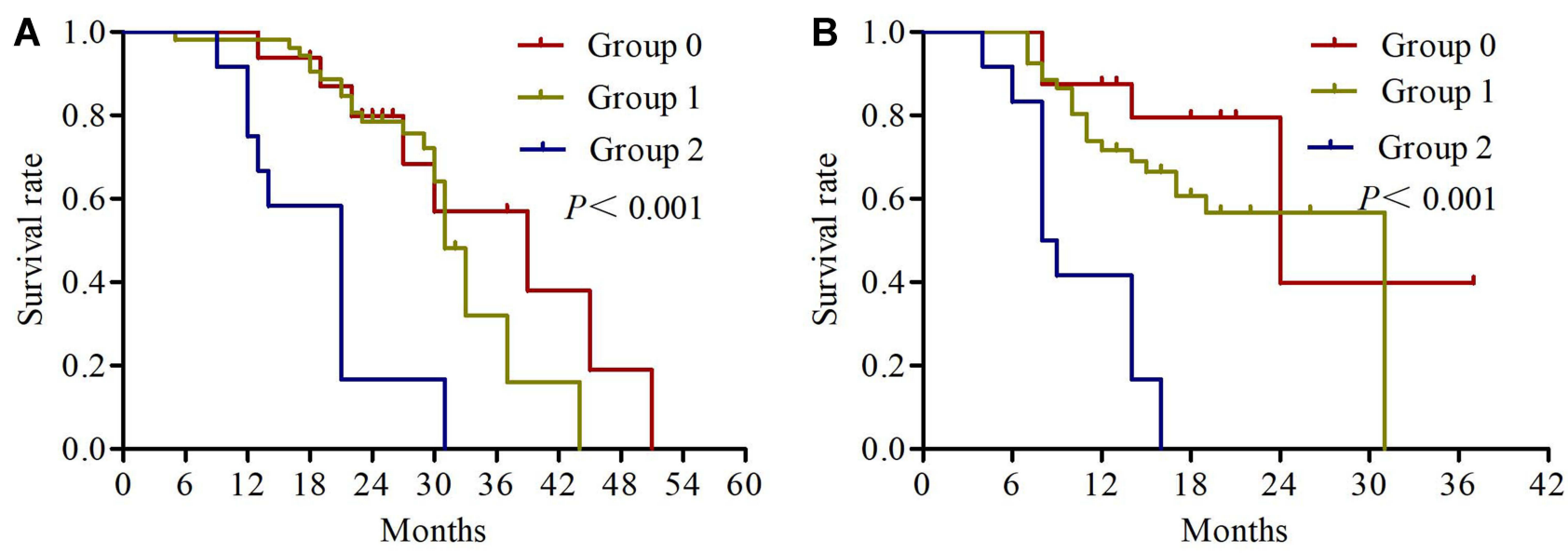

C

D
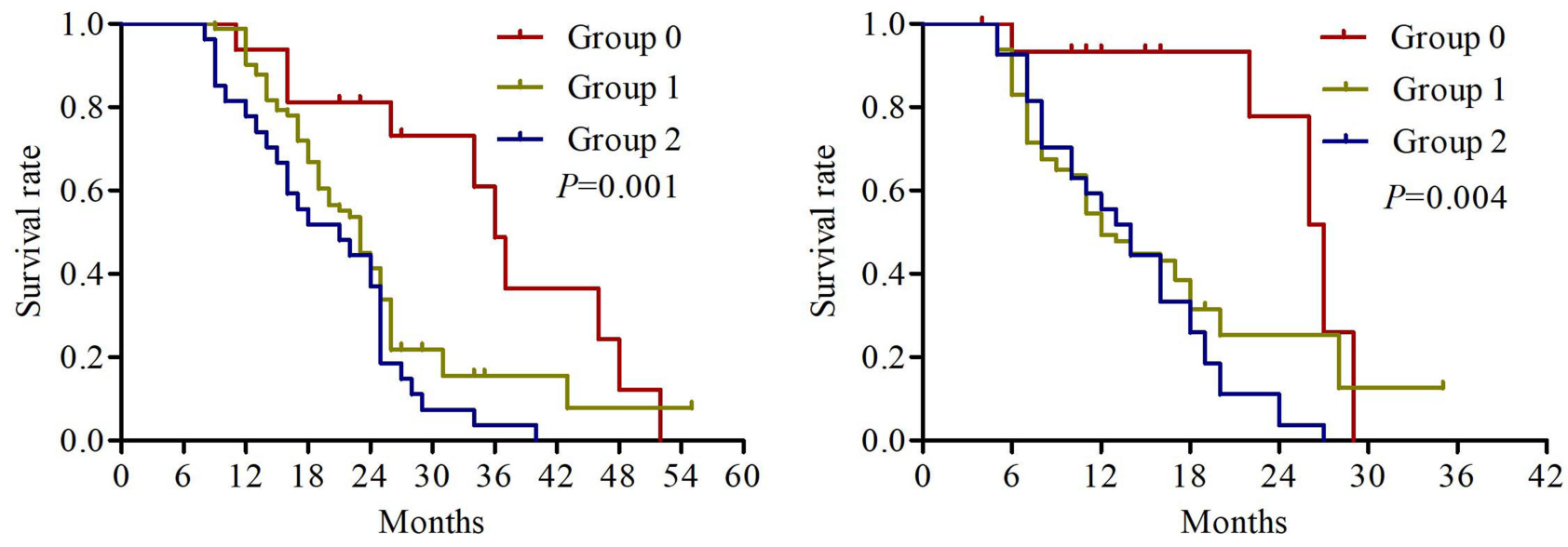

Figure 6 Kaplan-Meier survival curves of overall survival (A) and progression-free survival (B) for NPS group in stage IIIA NSCLC, and overall survival (C) and progressionfree survival (D) for NPS group in stage IIIB NSCLC.

than other prognostic systems. By summarizing these research conclusions, NPS is an easily available and comprehensive predictive evaluation method, which represents a patient's whole-systemic inflammation and nutritional status in multidimensional aspects. As we have studied, our results revealed that NPS had the largest AUC compared with other indicators (Serum albumin, total cholesterol, NLR and LMR) according to the ROC analysis. We further proposed a new prediction model based on NPS, and results revealed that NPS was a strong independent prognostic factor. In this regard, its clinical application value was highlighted, which indicated its superiorities for identified patients with higher risk of recurrence or mortality.

Some limitations need to be addressed in this study. First, our study is a retrospective study, with a relatively small sample from a single center, which may reduce the generalizability of the study results. At the same time, selection bias is inevitable because of study limitations. Second, laboratory indicators such as serum albumin, total cholesterol, neutrophils lymphocytes and monocytes may be influenced by various conditions, although we have strict inclusion criteria. Finally, the cutoff values of laboratory indicators were derived from the previous literature reports, which were not calculated by the highest Youden's index. This may not reflect differences in individual findings. Even so, the prognostic significance of NPS also presents considerable value in stage III NSCLC patients.

In this study, the NPS score system is an independent prognostic model affecting outcomes in stage III NSCLC patients. We found that the high NPS score is associated with poor OS and PFS. And the combination of laboratory indicators as NPS score system was superior to either indicator alone in terms of predicting ability. NPS score system can provide a more accurate assessment of outcomes and opinions on individualized hazard classification for stage III NSCLC patients. 
Table 4 Multivariate Cox Regression Analysis of OS and PFS

\begin{tabular}{|c|c|c|c|c|c|c|}
\hline \multirow[t]{2}{*}{ Characteristics } & \multicolumn{3}{|c|}{ os } & \multicolumn{3}{|c|}{ PFS } \\
\hline & HR & $95 \% \mathrm{Cl}$ & $P$-value & HR & $95 \% \mathrm{Cl}$ & P-value \\
\hline KPS & & & 0.436 & & & 0.710 \\
\hline $90-100$ & 1.000 & $0.668-2.548$ & & 1.000 & $0.5690-2.288$ & \\
\hline $70-80$ & 1.305 & & & 1.141 & & \\
\hline Differentiation & & & 0.74 & & & 0.58 \\
\hline Well/Moderate & 1.000 & $0.548-2.331$ & 0 & 1.000 & $0.578-2.638$ & 6 \\
\hline Poor & 1.130 & & & 1.235 & & \\
\hline Primary tumor size & & & 0.130 & & & \\
\hline$\leq 4 \mathrm{~cm}$ & 1.000 & $0.924-1.859$ & & & & \\
\hline$>4 \mathrm{~cm}$ & 1.310 & & & & & \\
\hline cTNM status & & & 0.03 & & & 0.07 \\
\hline IIIA & 1.000 & $1.038-2.204$ & 1 & 1.000 & $0.958-2.181$ & 9 \\
\hline IIIB & 1.512 & & & 1.446 & & \\
\hline Response rate & & & 0.189 & & & 0.092 \\
\hline$C R+P R$ & 1.000 & $0.885-1.856$ & & 1.000 & $0.944-2.155$ & \\
\hline$S D+P D$ & 1.282 & & & 1.426 & & \\
\hline NPS Group & & & 0.001 & & & 0.006 \\
\hline $0 / 1$ & 1.000 & $1.326-2.98 \mid$ & & 1.000 & I. $|82-2.72|$ & \\
\hline 2 & 1.988 & & & 1.793 & & \\
\hline
\end{tabular}

Abbreviations: OS, overall survival; PFS, progression-free survival; HR, hazard ratio; $\mathrm{Cl}$, confidence interval; KPS, Karnofsky performance score; CR, complete response; $\mathrm{PR}$, partial response; $\mathrm{SD}$, stable disease; $\mathrm{PD}$, progressive disease.

\section{Acknowledgments}

This study was supported by Grant (2020117) from the The Gusu Health Talent Program and Weifang Health Scientific research Project (WFWSJK-2021-003).

\section{Disclosure}

The authors report no conflicts of interest in this work.

\section{References}

1. Bray F, Ferlay J, Soerjomataram I, Siegel RL, Torre LA, Jemal A. Global cancer statistics 2018: GLOBOCAN estimates of incidence and mortality worldwide for 36 cancers in 185 countries. CA Cancer J Clin. 2018;68(6):394-424. doi:10.3322/caac.21492

2. Kishida Y, Hirose T, Shirai T, et al. Myelosuppression induced by concurrent chemoradiotherapy as a prognostic factor for patients with locally advanced non-small cell lung cancer. Oncol Lett. 2011;2 (5):949-955. doi:10.3892/ol.2011.348

3. Curran WJ Jr, Paulus R, Langer CJ, et al. Sequential vs. concurrent chemoradiation for stage III non-small cell lung cancer: randomized Phase III trial RTOG 9410. J Natl Cancer Inst. 2011;103 (19):1452-1460. doi:10.1093/jnci/djr325

4. Feng W, Fu XL, Cai XW, et al. Patterns of local-regional failure in completely resected stage IIIA(N2) non-small cell lung cancer cases: implications for postoperative radiation therapy clinical target volume design. Int $J$ Radiat Oncol Biol Phys. 2014;88(5):1100-1107. doi:10.1016/j.ijrobp.2013.12.048
5. Kong FM, Ten HRK, Schipper MJ, et al. High-dose radiation improved local tumor control and overall survival in patients with inoperable/unresectable non-small-cell lung cancer: long-term results of a radiation dose escalation study. Int J Radiat Oncol Biol Phys. 2005;63(2):324-333. doi:10.1016/j.ijrobp.2005.02.010

6. Machtay M, Bae K, Movsas B, et al. Higher biologically effective dose of radiotherapy is associated with improved outcomes for locally advanced non-small cell lung carcinoma treated with chemoradiation: an analysis of the Radiation Therapy Oncology Group. Int J Radiat Oncol Biol Phys. 2012;82(1):425-434. doi:10.1016/j. ijrobp.2010.09.004

7. Goldstraw P, Crowley J, Chansky K, et al. The IASLC Lung Cancer Staging Project: proposals for the revision of the TNM stage groupings in the forthcoming (seventh) edition of the TNM Classification of malignant tumours. $J$ Thorac Oncol. 2007;2(8):706-714. doi:10.1097/JTO.0b013e31812f3c1a

8. Mantovani A, Allavena P, Sica A, Balkwill F. Cancer-related inflammation. Nature. 2008;454(7203):436-444. doi:10.1038/ nature 07205

9. Sato H, Tsubosa Y, Kawano T. Correlation between the pretherapeutic neutrophil to lymphocyte ratio and the pathologic response to neoadjuvant chemotherapy in patients with advanced esophageal cancer. World J Surg. 2012;36(3):617-622. doi:10.1007/s00268-011-1411-1

10. Yang L, Karin M. Roles of tumor suppressors in regulating tumor-associated inflammation. Cell Death Differ. 2014;21 (11):1677-1686. doi:10.1038/cdd.2014.131

11. Guo D, Jin F, Jing W, et al. Incorporation of the SUVmax measured from FDG PET and neutrophil-to-lymphocyte ratio improves prediction of clinical outcomes in patients with locally advanced non-smallcell lung cancer. Clin Lung Cancer. 2019;20(6):412-419. doi:10.1016/j.cllc.2019.06.008 
12. Zhang N, Jiang J, Tang S, Sun G. Predictive value of neutrophil-lymphocyte ratio and platelet-lymphocyte ratio in non-small cell lung cancer patients treated with immune checkpoint inhibitors: a meta-analysis. Int Immunopharmacol. 2020;85:106677. doi:10.1016/j.intimp.2020.106677

13. Guo D, Zhang J, Jing W, et al. Prognostic value of systemic immune-inflammation index in patients with advanced non-smallcell lung cancer. Future Oncol. 2018;14(25):2643-2650. doi: $10.2217 /$ fon-2018-0285

14. Hua X, Long ZQ, Huang X, et al. The value of Prognostic Nutritional Index (PNI) in predicting survival and guiding radiotherapy of patients with T1-2N1 breast cancer. Front Oncol. 2019;9:1562. doi:10.3389/fonc.2019.01562

15. Yang C, Wei C, Wang S, et al. Combined features based on preoperative controlling nutritional status score and circulating tumour cell status predict prognosis for colorectal cancer patients treated with curative resection. Int J Biol Sci. 2019;15(6):1325-1335. doi:10.7150/ijbs.33671

16. Suzuki Y, Okabayashi K, Hasegawa H, et al. Comparison of preoperative inflammation-based prognostic scores in patients with colorectal cancer. Ann Surg. 2018;267(3):527-531. doi:10.1097/ SLA.0000000000002115

17. Li Q, Cong R, Wang Y, et al. Naples prognostic score is an independent prognostic factor in patients with operable endometrial cancer: results from a retrospective cohort study. Gynecol Oncol. 2021;160 (1):91-98. doi:10.1016/j.ygyno.2020.10.013

18. Galizia G, Lieto E, Auricchio A, et al. Naples Prognostic score, based on nutritional and inflammatory status, is an independent predictor of long-term outcome in patients undergoing surgery for colorectal cancer. Dis Colon Rectum. 2017;60(12):1273-1284. doi:10.1097/ DCR.0000000000000961

19. Feng JF, Zhao JM, Chen S, Chen QX. Naples prognostic score: a novel prognostic score in predicting cancer-specific survival in patients with resected esophageal squamous cell carcinoma. Front Oncol. 2021;11:652537. doi:10.3389/fonc.2021.652537

20. Miyamoto Y, Hiyoshi Y, Daitoku N, et al. Naples prognostic score is a useful prognostic marker in patients with metastatic colorectal cancer. Dis Colon Rectum. 2019;62(12):1485-1493. doi:10.1097/ DCR.0000000000001484

21. Balkwill F, Mantovani A. Inflammation and cancer: back to Virchow? Lancet. 2001;357(9255):539-545. doi:10.1016/S0140-6736(00)04046-0

22. Zhu H, Cao X. NLR members in inflammation-associated carcinogenesis. Cell Mol Immunol. 2017;14(5):403-405. doi: $10.1038 / \mathrm{cmi} .2017 .14$

23. Wang D, DuBois RN. Immunosuppression associated with chronic inflammation in the tumor microenvironment. Carcinogenesis. 2015;36(10):1085-1093. doi:10.1093/carcin/bgv123

24. Takahashi R, Mabuchi S, Kuroda H, et al. The SIGNIFICANCE of pretreatment thrombocytosis and its association with neutrophilia in patients with surgically treated endometrial cancer. Int $J$ Gynecol Cancer. 2017;27(7):1399-1407. doi:10.1097/IGC.0000000000001019

25. Schernberg A, Nivet A, Dhermain F, et al. Neutrophilia as a biomarker for overall survival in newly diagnosed high-grade glioma patients undergoing chemoradiation. Clin Transl Radiat Oncol. 2018;10:47-52. doi:10.1016/j.ctro.2018.04.002

26. Ray-Coquard I, Cropet C, Van Glabbeke M, et al. Lymphopenia as a prognostic factor for overall survival in advanced carcinomas, sarcomas, and lymphomas. Cancer Res. 2009;69(13):5383-5391. doi:10.1158/0008-5472.CAN-08-3845

27. Tang C, Liao Z, Gomez D, et al. Lymphopenia association with gross tumor volume and lung V5 and its effects on non-small cell lung cancer patient outcomes. Int J Radiat Oncol Biol Phys. 2014;89 (5):1084-1091. doi:10.1016/j.ijrobp.2014.04.025

28. Menetrier-Caux C, Ray-Coquard I, Blay JY, Caux C. Lymphopenia in cancer patients and its effects on response to immunotherapy: an opportunity for combination with cytokines? J Immunother Cancer. 2019;7(1):85. doi:10.1186/s40425-019-0549-5
29. Kitamura T, Doughty-Shenton D, Cassetta L, et al. Monocytes differentiate to immune suppressive precursors of metastasis-associated macrophages in mouse models of metastatic breast cancer. Front Immunol. 2017;8:2004. doi:10.3389/fimmu.2017.02004

30. Parikh K, Kumar A, Ahmed J, et al. Peripheral monocytes and neutrophils predict response to immune checkpoint inhibitors in patients with metastatic non-small cell lung cancer. Cancer Immunol Immunother. 2018;67(9):1365-1370. doi:10.1007/s00262018-2192-2

31. Houghton AM, Rzymkiewicz DM, Ji H, et al. Neutrophil elastase-mediated degradation of IRS-1 accelerates lung tumor growth. Nat Med. 2010;16(2):219-223. doi:10.1038/nm.2084

32. Wu L, Saxena S, Awaji M, Singh RK. Tumor-associated neutrophils in cancer: going pro. Cancers (Basel). 2019;11:4. doi:10.3390/ cancers 11040564

33. Droin N, Hendra JB, Ducoroy P, Solary E. Human defensins as cancer biomarkers and antitumour molecules. $J$ Proteomics. 2009;72(6):918-927. doi:10.1016/j.jprot.2009.01.002

34. Shankaran V, Ikeda H, Bruce AT, et al. IFNgamma and lymphocytes prevent primary tumour development and shape tumour immunogenicity. Nature. 2001;410(6832):1107-1111. doi:10.1038/ 35074122

35. Laviron M, Combadiere C, Boissonnas A. Tracking monocytes and macrophages in tumors with live imaging. Front Immunol. 2019;10:1201. doi:10.3389/fimmu.2019.01201

36. Bun A, Fujimoto Y, Higuchi T, et al. Prognostic significance of neutrophil-to-lymphocyte ratio in luminal breast cancers with low levels of tumour-infiltrating lymphocytes. Anticancer Res. 2020;40 (5):2871-2880. doi:10.21873/anticanres.14263

37. Templeton AJ, McNamara MG, Seruga B, et al. Prognostic role of neutrophil-to-lymphocyte ratio in solid tumors: a systematic review and meta-analysis. J Natl Cancer Inst. 2014;106(6):dju124. doi:10.1093/jnci/dju124

38. Zhao QT, Yang Y, Xu S, et al. Prognostic role of neutrophil to lymphocyte ratio in lung cancers: a meta-analysis including 7054 patients. Onco Targets Ther. 2015;8:2731-2738. doi:10.2147/OTT.S90875

39. Nishijima TF, Muss HB, Shachar SS, Tamura K, Takamatsu Y. Prognostic value of lymphocyte-to-monocyte ratio in patients with solid tumors: a systematic review and meta-analysis. Cancer Treat Rev. 2015;41(10):971-978. doi:10.1016/j.ctrv.2015.10.003

40. Yan H, Cai L, Chen S, Li J. Preoperative lymphocyte-to-monocyte ratio versus platelet-to-lymphocyte ratio as a prognostic predictor for non-small cell lung cancer patients. J Med Biochem. 2020;39 (2):160-164. doi:10.2478/jomb-2019-0031

41. Tokunaga R, Sakamoto Y, Nakagawa S, et al. CONUT: a novel independent predictive score for colorectal cancer patients undergoing potentially curative resection. Int J Colorectal Dis. 2017;32 (1):99-106. doi:10.1007/s00384-016-2668-5

42. Morhij R, Mahendra A, Jane M, McMillan DC. The modified Glasgow prognostic score in patients undergoing surgery for bone and soft tissue sarcoma. J Plast Reconstr Aesthet Surg. 2017;70 (5):618-624. doi:10.1016/j.bjps.2017.01.016

43. Sun H, Yuan Y, Wang $P$, et al. Intensified low-density lipoprotein-cholesterol target of statin therapy and cancer risk: a meta-analysis. Lipids Health Dis. 2015;14:140. doi:10.1186/s12944015-0147-6

44. Tanne JH. Meta-analysis says low LDL cholesterol may be associated with greater risk of cancer. BMJ. 2007;335(7612):177. doi:10.1136/ bmj.39287.415347.DB

45. Tomita M, Ayabe T, Shimizu T, Nakamura K. Preoperative total serum cholesterol and patients' survival in resected nonsmall cell lung cancer. Lung Cancer Int. 2012;2012:463520. doi:10.1155/ 2012/463520

46. Mandal CC, Rahman MM. Targeting intracellular cholesterol is a novel therapeutic strategy for cancer treatment. J Cancer Sci Ther. 2014;6(12):510-513. doi:10.4172/1948-5956.1000316 
47. Oliver MF. Serum cholesterol-the knave of hearts and the joker. Lancet. 1981;2(8255):1090-1095. doi:10.1016/S0140-6736(81)91286-1

48. Nakagawa N, Yamada S, Sonohara F, et al. Clinical implications of Naples prognostic score in patients with resected pancreatic cancer. Ann Surg Oncol. 2020;27(3):887-895. doi:10.1245/s10434-019-08047-7

49. Kano K, Yamada T, Yamamoto K, et al. The impact of pretherapeutic Naples prognostic score on survival in patients with locally advanced esophageal cancer. Ann Surg Oncol. 2021;28(8):4530-4539. doi:10.1245/s10434-020-09549-5
50. Li S, Wang H, Yang Z, et al. Naples Prognostic Score as a novel prognostic prediction tool in video-assisted thoracoscopic surgery for early-stage lung cancer: a propensity score matching study. Surg Endosc. 2021;35(7):3679-3697. doi:10.1007/s00464-02007851-7

\section{Publish your work in this journal}

The Journal of Inflammation Research is an international, peerreviewed open-access journal that welcomes laboratory and clinical findings on the molecular basis, cell biology and pharmacology of inflammation including original research, reviews, symposium reports, hypothesis formation and commentaries on: acute/chronic inflammation; mediators of inflammation; cellular processes; molecular mechanisms; pharmacology and novel anti-inflammatory drugs; clinical conditions involving inflammation. The manuscript management system is completely online and includes a very quick and fair peerreview system. Visit http://www.dovepress.com/testimonials.php to read real quotes from published authors.

Submit your manuscript here: https://www.dovepress.com/journal-of-inflammation-research-journal 